\title{
Difficulties of facelift in patients with scar after treatment of facial liposuction or thread lift sutures
}

\author{
Zhi-Jun Wang, MD, PhD ${ }^{1} \mathbb{1}$, Guanyi Li, $\mathrm{MD}^{1} \mathbb{1}$, Shuzhong Guo, MD, $\mathrm{PhD}^{2} \mathbb{D}$, DongQing Fu, $\mathrm{MD}^{3} \mathbb{1}$, \\ Bozhang Shen, $\mathrm{MD}^{3} \mathbb{1}$, Chengxin Bai, $\mathrm{MD}^{1}$ (iD) \\ 'Institute of Facial Rejuvenation, Plastic Surgery Hospital of Xi'an International Medical Center, Xi'an, China \\ ${ }^{2}$ Plastic Surgery Hospital Ear in Manufacturing Center, Xi'an International Medical Center, Xi'an, China \\ ${ }^{3}$ Medical School of Yanbian University, Ji Ling, China
}

Objective: To explore the methods and safety of general abnormal anatomical layers and structure in rhytidectomy, which is also of clinical significance. In this group, 39 patients with static and dynamic deformities formed at different levels after liposuction and thread technique more than one time were treated with stable and active separation skills in high SMAS facelift operation. The results showed that the patients of this group could get the same effect of younger postoperative operation as "initial face" and solve dynamic adhesion deformity.

Methods: Magnetic resonance imaging and ultrasound were performed before operation, focusing on the abnormal changes of facial soft tissue. The results showed that the signal of the foreign body, the position and texture of scar, the relationship between scar and masseter fascia, buccal fat pad, and whether there was tissue defect or hyperplasia. In the operation, the normal separation was made in the second and fourth layers of the previously safe and easy to separate plane, instead of in the dense one or two layers of the scar. It is an alternative microsurgical separation technique. The key point is to separate the incomplete SMAS flap to achieve the expected good effect of rhytidectomy, release the scar adhesion and facial spasm. At the same time, the severed injury of facial nerve branches should be strictly prevented.

Results: 74 cases of facelift were taken in recent eight months and 67 patients were treated with micro plastic surgery. Among them, 39 patients received more than one facial liposuction and more than one thread lift. In the scar formed by liposuction and thread technology, liposuction is especially serious; liposuction can still absorb the structural layer, and scar directly involves the SMAS and buccal fat pad envelope containing facial nerve branches, one of which directly causes the injury of the mandibular marginal branch. Most of the chronic granuloma scar formed after the degradation of the thread technology is in the facial space, which causes the scar atresia of the space. The flap and SMAS flap of 39 cases were separated, most of which were facial nerve branches in scar. All of them were difficult to separate and form the SMAS flap of superficial temporal fascia-Orbicularis oculi-platysma, and $89.6 \%$ of the excellent facelift effect was obtained. Facial nerve injury was found in 2 cases, 1 case was right mandible marginal branch traction injury, and the other side was left corner nerve injury. In the process of recovery.

Conclusion: For patients with facial liposuction and thread technique, doctors must be prepared for the difficult separation of scar adhesion and have a high risk of facial nerve branch disconnection. If we don't pursue the rejuvenation effect, it will lead to the existence of surgical effect discount, bilateral asymmetry, and dynamic adhesion deformity.

Keywords: aspiration lipectomy; cicatrix; facial nerve; rhytidoplasty; scar; sutures 


\section{Introduction}

The characteristics of facial anatomy are composed of delicacy, structure, and function. Additionally, the complexity of the upper nerve branch anatomy has served as a deterrent for the applying of facelift, embodying itself to become one of the most difficult operations in cosmetic surgery. Clinically, the case of facial liposuction has increased in the past one or two years, and in the recent five years, thread lift has been widely used. Among those patients, almost all have experienced thread lift treatment more than once.

After facial liposuction, some dense scar adhesions might be formed in the subcutaneous layer or even in the sub-SMAS layer. Also, the facial nerve branch and zygomatic muscle can adhere to the scar formed in the adjacent layer after thread lift. Undoubtedly, these two types of scars may pose an adverse impact, which is common in this group and significantly increases the risk of facial nerve branch injury, on dissecting SMAS in the facelift. If it is found that the masseter fascia and/or buccal fat pad capsule has been severely affected by the fibrotic scar during the operation, the risk of facial nerve branch injury is almost inevitable. This situation forces the author to summarize the cases to learn lessons from the experience, looking forward to reducing the risk of the facelift. As a result, the effect of facial rejuvenation will be longer and stabler.

As mentioned above, the key point expressed and discussed in this article is the abnormal anatomical structure related to the process of dissection and suspension in facelift surgery. The results and conclusions of this research are not repeatable, and they cannot even meet the basic requirements of "standard surgery". This requires that this article must objectively focus on the descriptions below.

1) What are the "consequences" caused by facial liposuction and thread lift on the facial anatomy hierarchy. 2) What does this "consequence" mean to the surgeons when performing facelift. 3) How does this consequence affect the effect of rhytidectomy? These three questions themselves have put serious tests on plastic surgeons. As for the "Panacea", the author regrets that it does not exist so far at the moment of writing this article.

\section{Materials and methods}

\section{Clinical information}

This research has selected 79 cases of high-facelift patients recruited by our hospital in the period of 1 year and 3 months from April 2020 to July 2021. The 24 cases without a history of facial liposuction or thread lift were excluded, and the remaining 55 cases were involved in the study. In the study group, 14 patients took both facial liposuction (abbreviated as liposuction) and thread-lift sutures (abbreviated as thread lift). Nine cases only had liposuction, and 32 cases only got thread lift. The cases of thread lift received an average of 1.5 times of treatment, and half of them received more than two times. Among them, the average age was 42 years (42.1 years); 29 cases were $29-40$ years old; 16 cases were 41-50 years old; and 10 cases were 51-61 years old. Twenty-nine cases were followed up, with the

Table 1. The average age is 42-year-old, 14-year-old, 46 person-times of thread lift, 23 person-times of facial liposuction, 25 person-times of facial fat grafting, 14 person-times of facelift, 27 person-times of filler injection

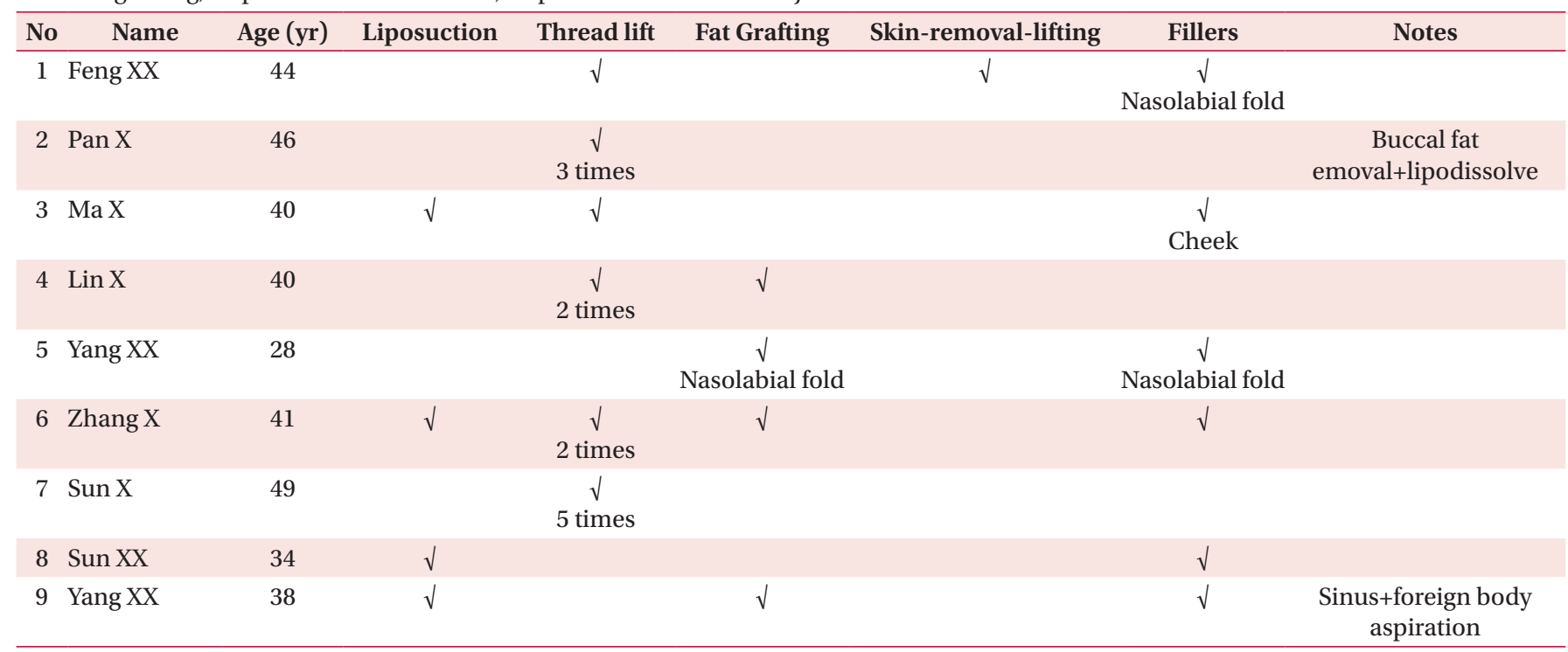


Table 1. Contnued 1

\begin{tabular}{|c|c|c|c|c|c|c|c|c|}
\hline No & Name & Age (yr) & Liposuction & Thread lift & Fat Grafting & Skin-removal-lifting & Fillers & Notes \\
\hline 10 & $\operatorname{LiX}$ & 39 & $\begin{array}{c}\sqrt{ } \\
2 \text { times }\end{array}$ & & $\sqrt{ }$ & & & \\
\hline 11 & Wang XX & 36 & $\begin{array}{c}\sqrt{ } \\
\text { Multiple }\end{array}$ & & $\begin{array}{c}\sqrt{ } \\
\text { Multiple }\end{array}$ & & $\begin{array}{c}\sqrt{ } \\
\text { Multiple }\end{array}$ & \\
\hline 12 & Chen XX & 57 & $\sqrt{ }$ & $\sqrt{ }$ & $\sqrt{ }$ & & & \\
\hline 13 & Song $\mathrm{X}$ & 43 & & & & & $\sqrt{ }$ & \\
\hline 14 & Zhang XX & 52 & $\sqrt{ }$ & $\begin{array}{c}\sqrt{ } \\
2 \text { times }\end{array}$ & & $\begin{array}{c}\sqrt{ } \\
3 \text { times }\end{array}$ & $\sqrt{ }$ & \\
\hline 15 & Huang XX & 29 & & & & & $\sqrt{ }$ & \\
\hline 16 & Wu XX & 37 & $\sqrt{ }$ & $\begin{array}{c}\sqrt{ } \\
3 \text { times }\end{array}$ & $\sqrt{ }$ & & & Hyaluronidase \\
\hline 17 & LiX & 61 & & $\sqrt{ }$ & & & & \\
\hline 18 & Yang XX & 37 & $\sqrt{ }$ & & $\sqrt{ }$ & & & \\
\hline 19 & Qi XX & 57 & & $\sqrt{ }$ & & & $\sqrt{ }$ & $\begin{array}{l}\text { More than } 10 \text { injections } \\
\text { of triamcinolone } \\
\text { acetonide }\end{array}$ \\
\hline 20 & Wang XX & 50 & & $\sqrt{ }$ & & & & \\
\hline 21 & Sun $\mathrm{X}$ & 34 & & $\sqrt{ }$ & & & $\sqrt{ }$ & \\
\hline 22 & Peng XX & 43 & & $\sqrt{ }$ & & & $\sqrt{ }$ & \\
\hline 23 & Zhao XX & 34 & & $\sqrt{ }$ & & & $\sqrt{ }$ & \\
\hline 24 & Luo XX & 38 & & $\begin{array}{c}\sqrt{ } \\
2 \text { times }\end{array}$ & & & & \\
\hline 25 & Zhang X & 36 & & $\sqrt{ }$ & & & $\begin{array}{c}\sqrt{ } \\
\text { Multiple }\end{array}$ & Hyaluronidase \\
\hline 26 & $\mathrm{Ll} \mathrm{XX}$ & 48 & & $\sqrt{ }$ & $\sqrt{ }$ & & & \\
\hline 27 & Ding $X$ & 56 & & $\sqrt{ }$ & & & $\sqrt{ }$ & \\
\hline 28 & Tan XX & 30 & & $\begin{array}{c}\sqrt{ } \\
3 \text { times }\end{array}$ & & & $\begin{array}{c}\sqrt{ } \\
2 \text { times }\end{array}$ & \\
\hline 29 & Yuan XX & 64 & & $\sqrt{ }$ & & & & Buccal fat removal \\
\hline 30 & Wang XX & 36 & $\sqrt{ }$ & $\begin{array}{c}\sqrt{ } \\
2 \text { times }\end{array}$ & $\sqrt{ }$ & & & Buccal fat removal \\
\hline 31 & Shi XX & 60 & & $\begin{array}{c}\sqrt{ } \\
2 \text { times }\end{array}$ & & & & \\
\hline 32 & Shi XX & 30 & & $\sqrt{ }$ & $\begin{array}{c}\sqrt{ } \\
2 \text { times }\end{array}$ & & & \\
\hline 33 & Shen XX & 49 & & $\sqrt{ }$ & $\sqrt{ }$ & & & \\
\hline 34 & $\mathrm{Xu} X$ & 39 & & $\sqrt{ }$ & & & $\sqrt{ }$ & $\begin{array}{c}\text { Lipodissolve injection } \\
3-5 \text { times }\end{array}$ \\
\hline 35 & Huang XX & 33 & & $\sqrt{ }$ & $\sqrt{ }$ & & $\sqrt{ }$ & Hyaluronidase \\
\hline 36 & YuX & 30 & $\sqrt{ }$ & & & & $\sqrt{ }$ & Buccal fat removal \\
\hline 37 & Huang Xx & 34 & & $\sqrt{ }$ & $\sqrt{ }$ & $\begin{array}{c}\sqrt{ } \\
\text { Forehead }\end{array}$ & $\sqrt{ }$ & $\begin{array}{l}\text { Buccal fat } \\
\text { removal+forehead } \\
\text { foreign }\end{array}$ \\
\hline 38 & $\mathrm{XuX}$ & 61 & $\sqrt{ }$ & & & $\sqrt{ }$ & & \\
\hline 39 & Yan X & 46 & & $\sqrt{ }$ & $\sqrt{ }$ & $\sqrt{ }$ & & Temporal fascia filling \\
\hline 40 & Zhuang X & 52 & $\sqrt{ }$ & $\begin{array}{c}\sqrt{ } \\
3 \text { times }\end{array}$ & & $\sqrt{ }$ & $\begin{array}{c}\sqrt{ } \\
\text { Mutiple }\end{array}$ & $\begin{array}{c}\text { Buccal fat } \\
\text { removal+lipodissolve }\end{array}$ \\
\hline 41 & $\operatorname{LiX}$ & 36 & & $\sqrt{ }$ & $\sqrt{ }$ & & $\begin{array}{c}\sqrt{ } \\
\text { Chin }\end{array}$ & $\begin{array}{l}\text { Intraoral foreign body } \\
\text { removal }\end{array}$ \\
\hline 42 & ChengX & 42 & $\begin{array}{c}\sqrt{ } \\
2 \text { times }\end{array}$ & $\sqrt{ }$ & $\sqrt{ }$ & & & $\begin{array}{c}\text { Buccal fat } \\
\text { removal+lipodissolve }\end{array}$ \\
\hline 43 & Tan XX & 41 & $\begin{array}{c}\sqrt{ } \\
3 \text { times }\end{array}$ & $\begin{array}{c}\sqrt{ } \\
2 \text { times }\end{array}$ & & & $\begin{array}{c}\sqrt{ } \\
\text { Nasolabial fold }\end{array}$ & \\
\hline
\end{tabular}


Table 1. Continued 2

\begin{tabular}{|c|c|c|c|c|c|c|c|c|}
\hline No & Name & Age (yr) & Liposuction & Thread lift & Fat Grafting & Skin-removal-lifting & Fillers & Notes \\
\hline 44 & Wang XX & 48 & & $\begin{array}{c}\sqrt{ } \\
3 \text { times }\end{array}$ & & & $\sqrt{ }$ & \\
\hline 45 & Guo X & 32 & & $\sqrt{ }$ & & & & $\begin{array}{l}\text { Facial lipodissolve } \\
\text { injection }\end{array}$ \\
\hline 46 & Zhou XX & 33 & $\sqrt{ }$ & $\sqrt{ }$ & $\sqrt{ }$ & & & \\
\hline 47 & GeX & 37 & $\begin{array}{c}\sqrt{ } \\
\text { Facelift }\end{array}$ & $\begin{array}{c}\sqrt{ } \\
2 \text { times }\end{array}$ & $\begin{array}{c}\sqrt{ } \\
2 \text { times }\end{array}$ & & $\begin{array}{c}\sqrt{ } \\
\mathrm{PLA}+\mathrm{HA}\end{array}$ & \\
\hline 48 & $\operatorname{Lin} X$ & 44 & & $\begin{array}{c}\sqrt{ } \\
2 \text { times }\end{array}$ & $\sqrt{ }$ & $\sqrt{ }$ & & \\
\hline 49 & Rong XX & 52 & & $\sqrt{ }$ & & & & \\
\hline 50 & Luo XX & 31 & $\sqrt{ }$ & $\begin{array}{c}\sqrt{ } \\
4-5 \text { times }\end{array}$ & $\sqrt{ }$ & & $\sqrt{ }$ & Lipectomy injection \\
\hline 51 & Zou XX & 32 & $\sqrt{ }$ & & $\sqrt{ }$ & $\sqrt{ }$ & & \\
\hline 52 & Jiang XX & 33 & & $\begin{array}{c}\sqrt{ } \\
3 \text { times }\end{array}$ & & & & \\
\hline & Ye X & 45 & & $\sqrt{ }$ & & & & \\
\hline 54 & Jiang XX & 42 & & $\sqrt{ }$ & $\begin{array}{c}\sqrt{ } \\
3 \text { times }\end{array}$ & & & \\
\hline & Zhou XX & 36 & & $\begin{array}{c}\sqrt{ } \\
2 \text { times }\end{array}$ & & & $\sqrt{ }$ & \\
\hline
\end{tabular}

longest follow-up period of 14 months and the shortest period of 3 months.

Some cases in this article also had other treatments before surgery, such as facial fat grafting, skin-removal-lift without dissecting SMAS, fillers injection, and so on. These factors are not included in the study of this research (Table 1).

In addition, each patient was given an opportunity to review the manuscript and consented to its publication.

\section{Procedures}

\section{Facelift surgical procedure}

In this article, the basic surgical procedures are divided into platysma plastic on the neck and High SMAS Facelift on the face. During the operation, the platysma muscle and the lateral half of the orbicularis oculi muscle were treated by "turning descending muscles into levator muscles".

Platysma plasty: After the superficial surface of the platysma muscle is dissected (the range is down to the first cervical striation, which is slightly larger to break the connection between the muscle and the dermis). Cut off the excess subcutaneous fat under direct vision. In most cases, they cannot be removed by liposuction. Then cut the front edge of the platysma muscle at the midline, and separate the platysma flaps on both sides in the gap between the deep surface of the platysma muscle and the superficial surface of the cervical fascia propria until the

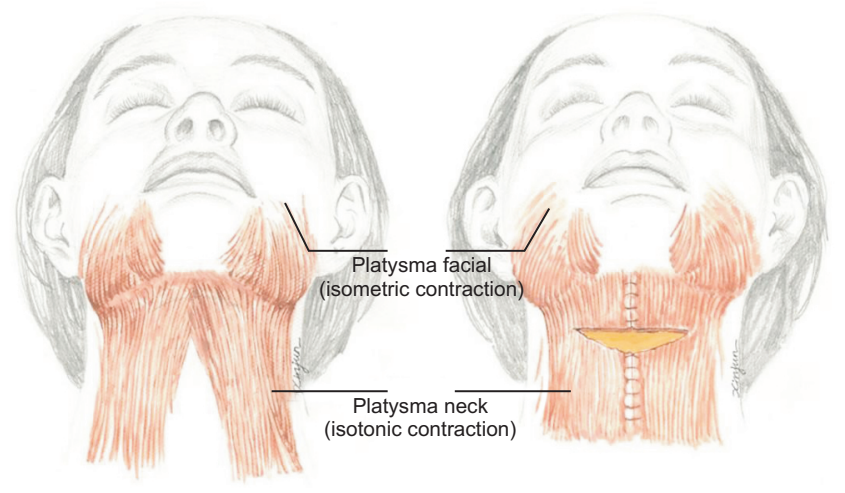

Fig. 1. Platysma plasty.

bilateral submandibular glands are exposed and stopped. After removing the excess platysma muscle by pulling it to the midline, suture the anterior edge of the full-length platysma longitudinally with appropriate tension. After suturing, the platysma is stretched from the deep plane. At the tightest point, usually, the hyoid bone is horizontal, a full-thickness incision is made to the platysma. The length is about $4-5 \mathrm{~cm}$-the platysma falls back and the neck thinning (Fig. 1).

Whether to get involved with the digastric muscles and submandibular glands depends on the situation.

Neck surgery is a combination of local anesthesia and regional nerve block, such as the mental nerve of the mental foramen, the transverse cervical nerve at the midpoint of the posterior 


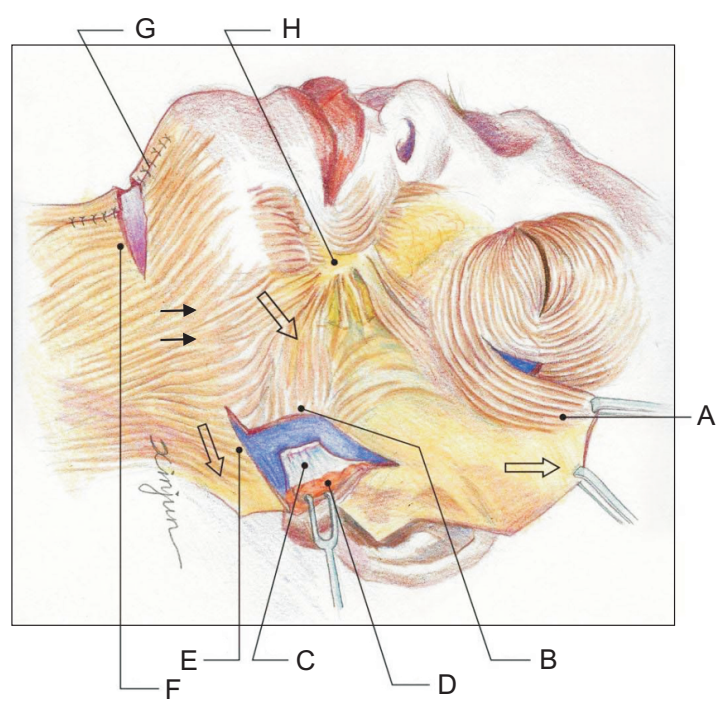

Fig. 2. The refined high SMAS facelift technique by author. (A) The lateral flap of the denervated orbicularis oculi muscle is tightened. (B) The transverse platysma muscle is lifted up backwards and fixed on the edge of the platysma-auricular ligament. (C, D) The triangular dense area on the deep surface of the earlobe. (E) Tighten the platysma skin flap fixed along the jawline. (F) Cut the platysma muscle along the plane of the hyoid bone. (G) Suture the medial edge of the platysma longitudinally. $(\mathrm{H})$ The modiolus is lifted.

edge of the sternocleidomastoid muscle, and so on.

\section{The basic high SMAS facelift}

The basic method of the author's SMAS facelift is Barton's high SMAS facelift [1]. Based on this basis, we have the following three improvements.

1) Completely lift and sever the lateral flap of the orbicularis oculi muscle to enter its inner facial nerve branch [2].

2) Cut off the platysma muscles horizontally in the middle and the outside, leaving a small amount of "pedicles" in the middle. After the lateral transverse dissection (approximately $4-6 \mathrm{~cm}$ ), as the lateral orbicularis oculi muscle flap-platysma flap is evenly lifted, the lateral orbicularis oculi flap [3] is fixed on the deep temporal fascia and. The transected upper edge of the platysma muscle is lifted obliquely and fixed on the broken edge of the platysmaauricular ligament (Fig. 2) [4]. Hereby, it is lifted and fixed and has the function of lifting the modiolus [3,4].

3) In most cases, the SMAS is lifted, showing that the bottom of the facial space is uneven and seldom flat: the parotid gland, accessory parotid gland, and buccal fat pad need to be treated to make it look as flat as possible (Fig. 3). If botulinum toxin is injected into the area, the obvious effect could be detected after 1 to 2 months. Normally, the pa-

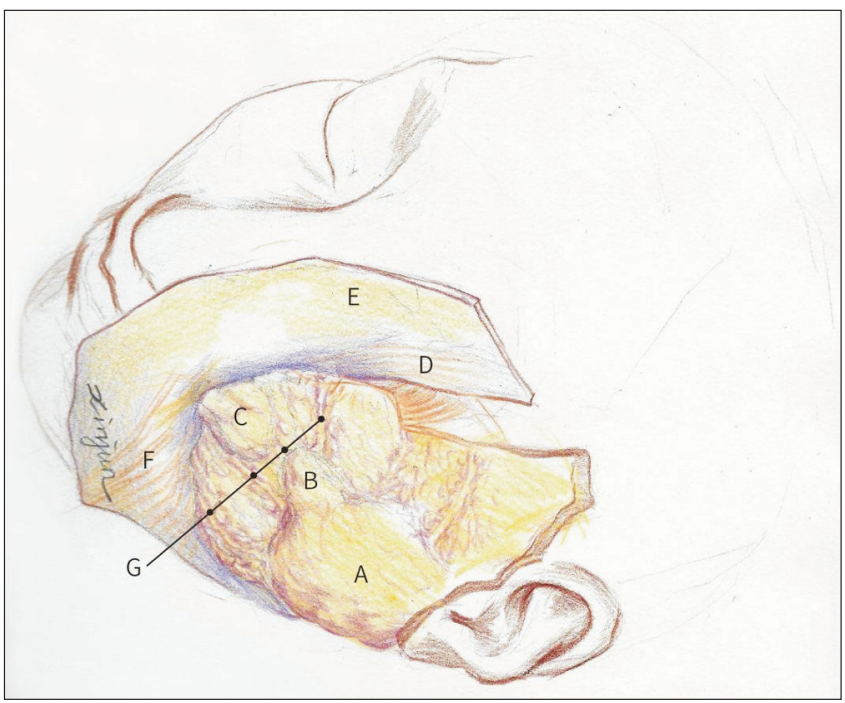

Fig. 3. The anatomy of deeper structures. (A) Enlarged sagging parotid gland; (B) accessory parotid gland; (C) buccal fat pad; (D) orbicularis oculi muscle; (E) superficial temporal fascia of SMAS; (F) latissimus cervicalis; (G) hidden in a translucent thin bite. Facial nerve branch within the myofascial membrane.

rotid gland and the buccal fat pad are protruding a lot. This is one of the reasons for the "bigger face aging" in which the aging face is broad and extended. Reducing these sagging, bulging, and hyperplastic (parotid-accessoy parotid) structures are one-half of the strategy for turning a big face into a small face. This proves that in facelift, liposuction cannot be performed like subcutaneous fat hyperplasia of the abdomen.

\section{Dissection technique of cases after facial liposuction}

This type of patient has severe adhesions, so all the adhesions must be released; the scar hyperplasia after blunt liposuction injury is particularly obvious, so the dissection is difficult. For those cases where the scar is hard and does not have the normal hierarchy, separation under SMAS is the process of separating the branches of the facial nerve within the scar. The dissection of this type of patient has three key points: scissors, passing under direct vision, protecting the masseter fascia, and buccal fat pad capsule.

The author originally used special broad-headed tissue scissors to subcutaneously separate the subcutaneous layer, as known as undermining. However, when dissecting the postliposuction area, it is best to dissect sharply and bluntly under direct vision. First of all, I am worried that it will damage the subdermal vascular network if it is shallow, and it will damage the masseter fascia or buccal fat pad capsule when it is deep 
because there are branches of the facial nerve in the masseter fascia and buccal fat pad capsule [5]. The adhesion scar after liposuction could be dissected by sharp tissue scissors with a flat tip to cut the scar. Otherwise, it is more likely to slip to the soft non-scar SMAS plane. The post-liposuction scar, which is, closer to the dermis, the more compact and tougher the scar, while the platysma-orbicularis oculi muscle SMAS is soft muscle tissue except for the "covering" [2] on the deep and shallow surface. Therefore, the scissors in the dense scar between the dermis and SMAS can easily slide towards SMAS. In half of the cases, SMAS has been mostly aspirated (Fig. 4). The deep side of the scar is closely attached to the masseter fascia and buccal fat pad capsule. This greatly increases the risk of injury to the deep surface of the masseter fascia, buccal fat pad capsule, buccal branch of the facial nerve, and marginal mandibular branch when dissecting the subcutaneous scar [5]. At the same time, there is no possibility of an intact SMAS flap form because the SMAS is incomplete and full of large and small defects. Therefore, it is recommended to use eye scissors and the surgeon needs to wear a magnifying glass. Using sharp dissection on the side of the dense scar (in fact, blunt dissection is not feasible); when separating masseter muscle fascia and buccal fat pad capsule, our consistent method is to "make good use of blunt dissection".

\section{Dissection technique of postoperative cases with thread lift}

In this group of 32 patients with thread lift, only 1 case had the thread, and its scar passed the full length of the subcutaneous fat layer, and the remaining 31 cases had the thread and its scar through the temporal space and the anterior masseter space, anterior zygomatic space, and cheek space $[6,7]$. The dissection plane of the superficial temporal fascia-orbicularis

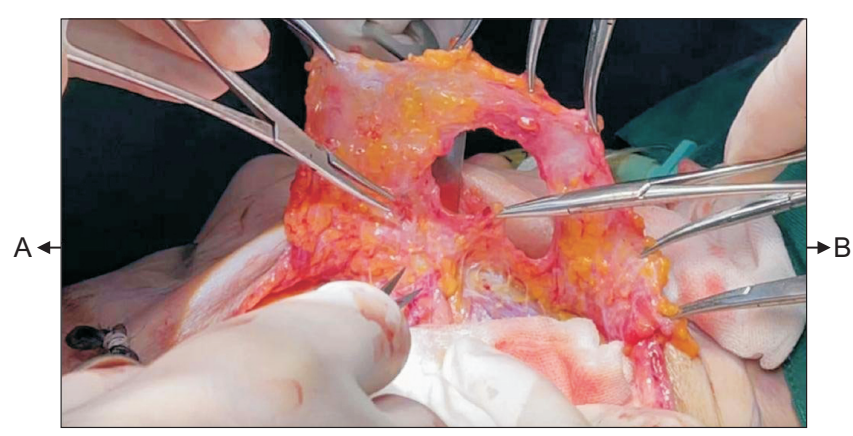

Fig. 4. The SMAS is damaged after facial liposuction (right side). The part of the SMAS pinched during the operation of which between the orbicularis oculi muscle and the platysma has been removed by liposuction, forming a large defect (A: cranial side; B: caudal side). muscle-platysma flap [8] of the high SMAS facelift is the abovementioned 4 facial spaces. The scar formed by the thread degradation of thread lift is dense and tough. Importantly, scars often involve masseter fascia and buccal fat pad capsules, which also cause mottled and intermittent scar adhesion. Because the facial nerve branches are in the masseter fascia and buccal fat pad capsule, each facial nerve branch may be involved by scars [5]. It was supposed to be an easy task to lift the huge SMAS flap (superficial temporal fascia-lateral orbicularis oculi-platysma muscle) from top to bottom on the sliding plane. However, it seems to be a very difficult ordeal to dissect the SMAS attributed to the severe scar adhesion of the facial nerve branch. If the scar area is large and the texture is tough, it might be easier to separate the tissue after using a high-pressure syringe to inject the tumescent fluid into the specific position.

\section{Results}

\section{Dissection time}

The dissection time of the SMAS flap for the facelift after liposuction is increased from the original normal time of 6-8 minutes/side to 30-60 minutes/side. While the dissection time after thread lift has increased drastically from the original average of 20 minutes/side to an average of 1.5 hours/side. Among those cases, the longest duration was 3 hours because the fragmented SMAS need sewing and patching up continuously (Fig. 5).

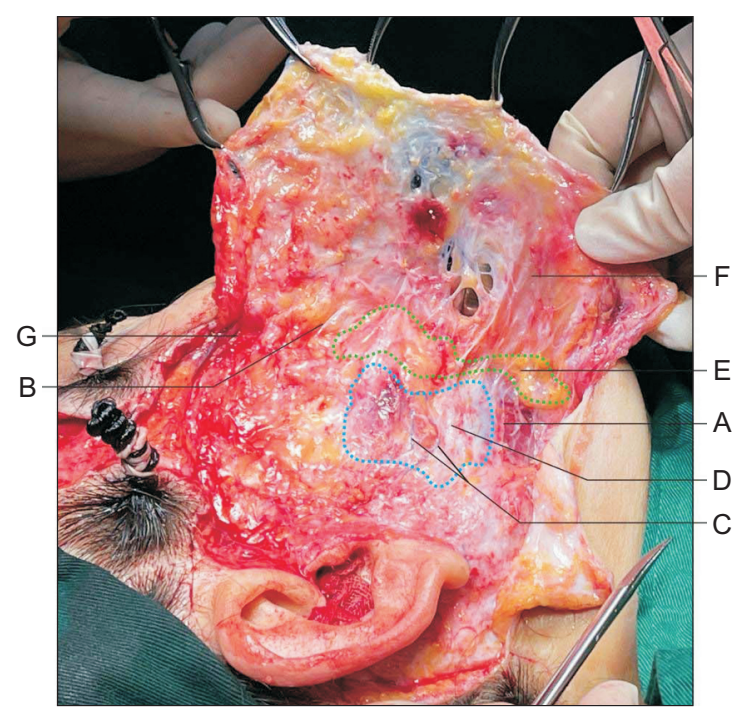

Fig. 5. Right side. During the dissection of the SMAS flap. (A) Marginal mandibular branch of facial nerve. (B) Maxillary branch of facial nerve. (C) Suture from thread lift. (D) Scarred masseter fascia. (E) Scarred buccal fat capsule. (F) Fragmented SMAS flap. (G) Zygomaticus. 
The stitching of the SMAS flap leaves two hidden drawbacks: 1) SMAS is not easy to be spread and tighten evenly by the surgeon as it is not a symmetrically curved surface because the viscoelasticity of the flap has been changed after stitching; 2) the surface skin is prone to unevenness, which is more likely to occur in patients with thinner skin.

\section{The outcome of the facelift}

From the author's perspective, there are three standards to evaluate the facelift outcomes-one is the clear contour, the second is the smooth lines, and the third is that the big face turns into a small face. Specifically, 6 points for clear contour (3 points for the clear jawline, 3 points for Ogee curve in S-shape); smooth lines: 3 points (it means that the aging problems have been solved, such as Indian patterns, nasolabial folds, and jowls. The facial lines are full and smooth at front view.); 1 point for a big face turn to small face (remove excess tissue as much as possible, especially for cases of unsightly face shape. Advance

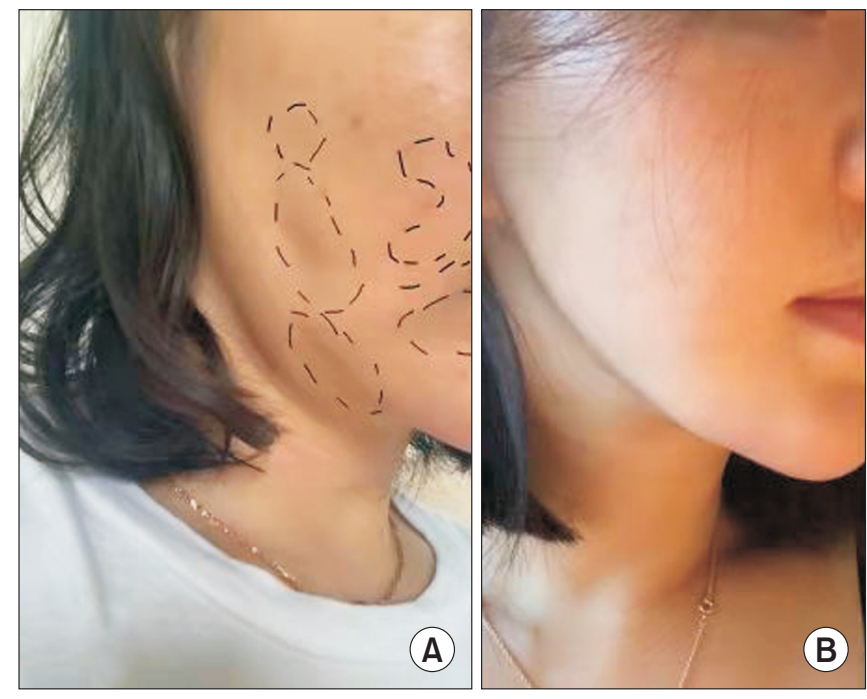

Fig. 6. A 38-year-old female, receiving high-SMAS facelift after facial liposuction. (A) The right face before high-SMAS facelift, and there are many depressed deformities in the mark. One of the depressions at the corner of the mouth is a dynamic deformity. (B) Ten-month postoperative.
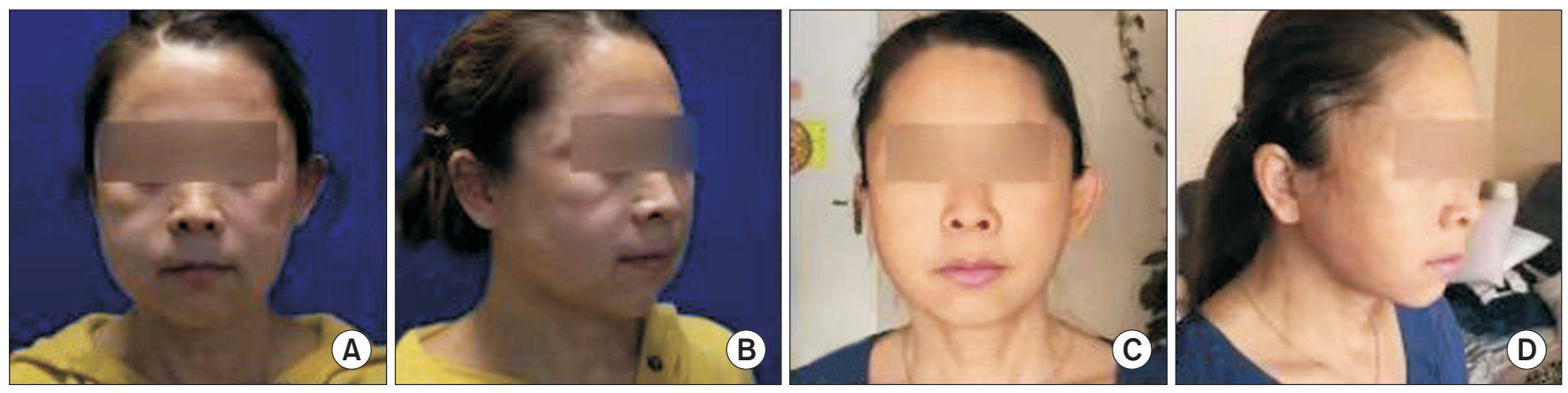

Fig. 7. A 52-year-old female, receiving the High-SMAS facelift after facial liposuction. (A, B) Preoperative, uneven deformities on both sides; (C, D) one-year postoperative.
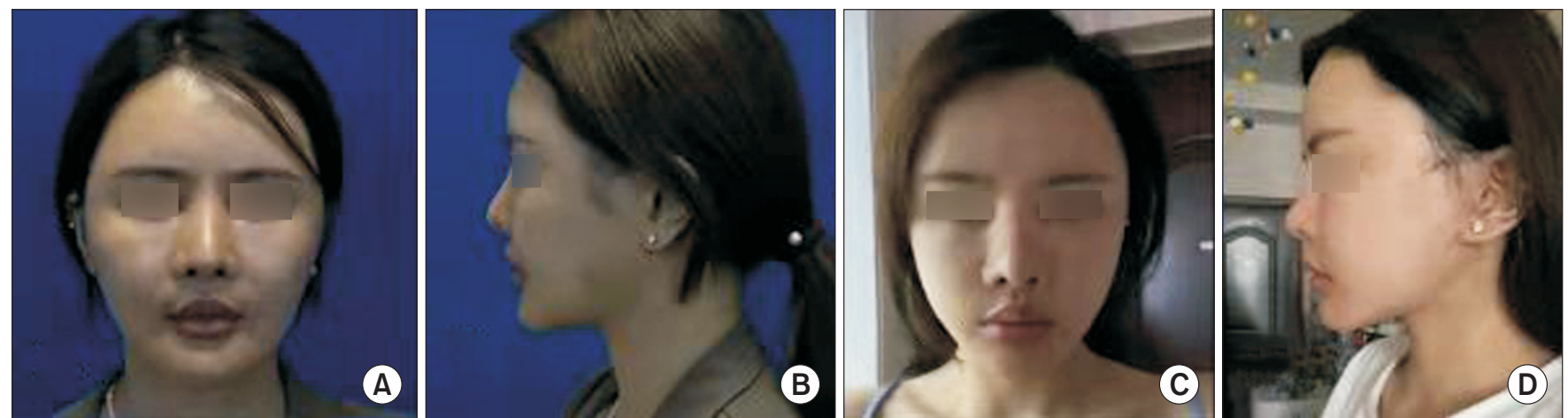

Fig. 8. A 31-year-old female, receiving high-SMAS facelift after facial liposuction and thread lift; (A, B) show preoperative slight dynamic and static deformities of the face due to aspiration lipectomy and cicatrix; (C, D) are high-SMAS face lift one and a half years after surgery, showing a rejuvenation effect and improvement of depression deformity. During the operation, there were extensive fibrotic scars in subcutaneous fat and facial space. 
facial tissue and change the aging-non-egg-shaped face to rejuvenated-egg-shaped face. In general, it is crucial to correct the enlarged and proliferated parotid glands, accessory parotid glands, and masseter muscles, and at the same time refill the temporal zone with the sagging SMAS flap).

There are 55 cases in the group, 29 cases were followed up. Among them, 7 cases $(24.1 \%)$ got 10 points, 19 cases (65.5\%) got 9 points, and 3 cases $(10.3 \%)$ got 8 points. In addition to the facial rejuvenation, the static and dynamic deformities caused by liposuction and thread lift before the operation have been effectively treated (Fig. 6-8).

\section{Facial nerve injury}

There were two cases of facial nerve injury, one was the right marginal mandibular branch, and the other was the left angular nerve. It shows that the right lower lip muscle is weak after the operation, and the contraction of the left corrugator supercilii muscle disappears. The two cases of facial nerve branch injury recovered at 3 and 6 months after surgery (Fig. 9).

\section{Discussion}

\section{The author's notion of the facelift}

The author believes that the meaning of facelift is aimed at the anatomical changes of the sagging aging structures, and achieves anatomical rejuvenation after surgery $[9,10]$. For example: 1) The aggravating of nasolabial folds is caused by the laxity of the zygomatic fat pad located under the skin $[11,12]$. Therefore, it is necessary to release the and the zygomatic retaining ligament and SMAS-malar ligament as much as possible to maximize the release of the deep entanglement of the zygomatic fat pad, to truly lift the mid-face and lessen the appearance of nasolabial folds [4]. At the same time, an ideal Ogee curve

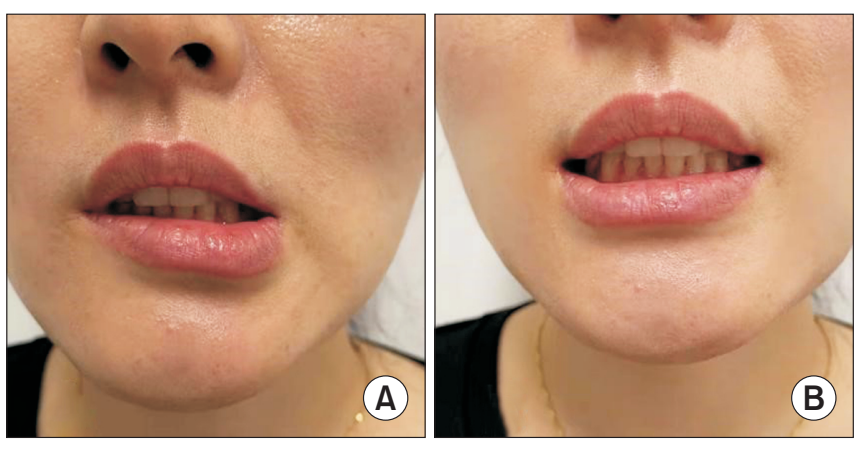

Fig. 9. A 40-year-old female, marginal mandibular branch injury after facial liposuction. (A) Facelift postoperative 15 days; (B) postoperative 3 months. Basically recovered.
[13] is obtained. The author believes that only the high SMAS technique can solve the aging problem of mid-face in Chinese patients [11]. 2) Based on the author's observation, evidence could be found that jowls are created by 4 anatomical reasons: (1) the angle of platysma tilts [14,15]; (2) the modiolus sags [14]; (3) the granule of subcutaneous fat near the modiolus is slightly being hypertrophic; (4) the buccal fat pad behind the modiolus is less resilient [14]. Therefore, it is necessary to completely dissect the severely sagging and straightened platysma, and at the same time release the platysma suspensory ligament [4] and the SMAS-malar ligament [4]. After all these ligaments are released, the buccal fat pad will be herniated. Then lay the fat pat flat and suspend it; lift the dissected platysma muscle at the mandibular edge back and upward, and suture it in the triangular dense area of the deep plane of earlobe [4]. Finally, reconstruct the platysma-auricular ligament to correct the angle of the platysma. If the dissection is sufficient, lifting of the platysma can lift the modiolus, resulting in improving jowls and jawline (Fig. 2). 3) After the procedures of maneuver parotid gland and accessory parotid gland, the orbicularis oculi-platysma-zygomatic fat pad, which aged earliest and sagging most, is lifted, flattened, and fixed. In addition, the degree of lifting, whether the surface is smooth or not, all depend on whether the flap is completely dissected from its both sides.

Above all, the author's plan of the facelift can be summarized as follow. Firstly, dissect the flap properly and completely. Secondly, reposition and suspend the flap correctly. Finally, always focus on the three major goals of facial rejuvenation. In this process, the anatomy of the facial nerve branch plays an important role as an anatomical landmark, which indicates the surgical entrance and high-risk zone $[5,16]$.

A $\quad$ B $\quad$ C $\quad$ D $\quad E$

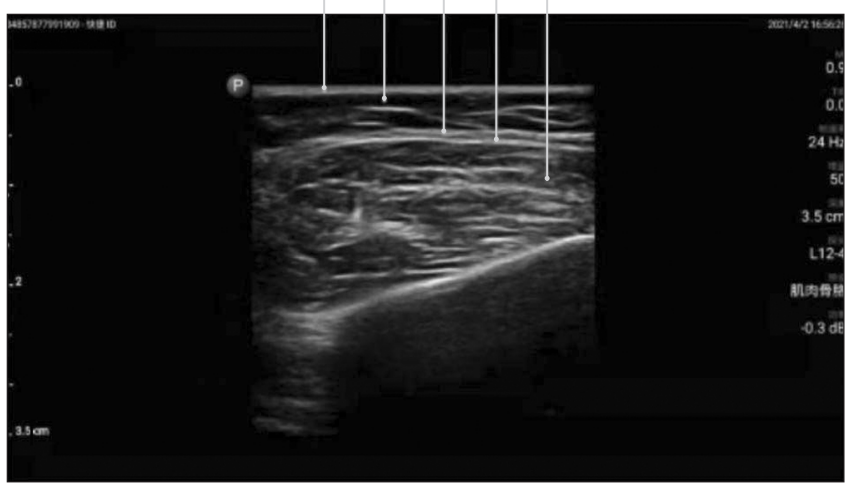

Fig. 10. A 34-year-old female, a normal ultrasonographic figure of facial soft tissue. (A) Skin; (B) subcutaneous fat; (C) SMAS; (D) masseter fascia; and (E) masseter muscle. 


\section{Facial liposuction scar and its effect on normal anatomical} structure

The author believes that one of the anatomical characteristics of facial soft tissue itself is tremendously delicate because of the exquisite layers, the exquisite structures, and the exquisite functions, which formed in long-term evolution. One of the downsides of facial liposuction is that because it is operated under blind vision, to some extent, ignores the exquisiteness of these hierarchical structures: whether it is the subcutaneous fat of the face itself, or the adjacent dermis and SMAS. These three layers are attached, thin and delicate (Fig. 10). The certain function of the three structures differs widely in different parts. As a result, when performing liposuction under blind vision, in addition to the damage to the hierarchy, structure, and function caused by non-targeted maneuvers, but also due to the large and dense subcutaneous fat pad, and the small diameter of the cannula and the tiny holes on it, the suction volume of fat is extremely low in most cases.

How to better perform facial liposuction? Here are the tips from the author: 1) It is best to use a flat cannula with a smaller diameter [1,9]; 2) Using preoperative ultrasound examination to locate and quantify the subcutaneous fat of the face and neck, obtaining the necessary preoperative diagnosis basis is an integral part of preplatysmal defatting. 3) The author agrees and recommends open-approach fat reduction surgery $[1,9]$. Such a precise method is in line with the delicate anatomical features of the face and the principle of protection in plastic surgery.

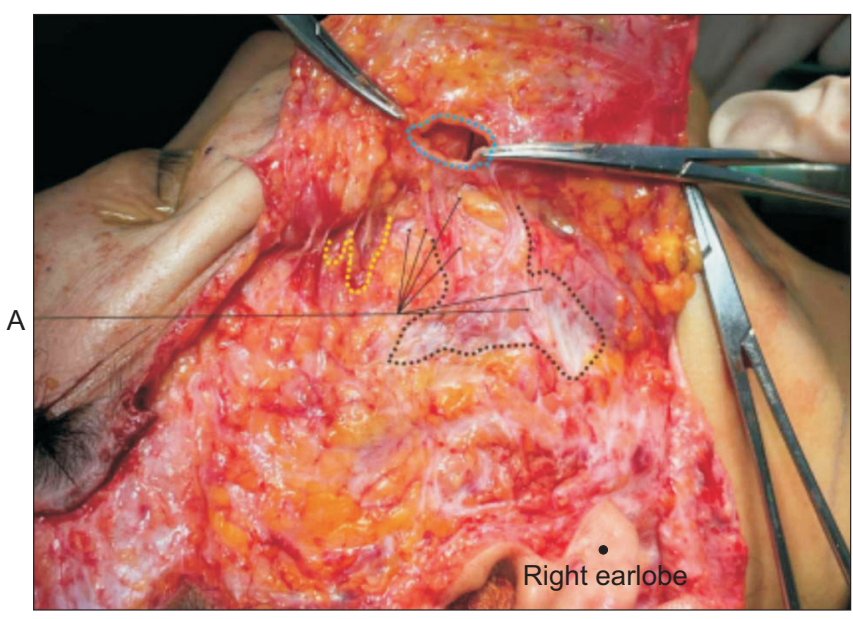

Fig. 11. Facelift on the post-thread-lift patient (right side). Blueline: damaged SMAS. Blackline: fibrosis masseter fascia and buccal fat pad capsule. Yellow line: zygomatic major and zygomatic minor. A, branch of the facial nerve in the scar.
Scar and its effect on the hierarchical structure of normal tissues after thread lift

Clinically, the long suture of thread lift sutures is most commonly inserted into the fascial space, which is the wrong plane. This situation is probably due to the ease of operation at this loose plane. However, the compatibility of various types of threads is obviously different in which worse ones might lead to mottled scars. In particular, the masseter fascia and buccal fat pad capsule containing the secondary and tertiary fibers of the facial nerve $[5,16]$ are either fibrotic, scarred or affected by scars (Fig. 11). In the high SMAS technique, it is necessary to dissect the superficial temporal fascia-orbicularis muscle-platysma flap in several facial spaces. The facial spaces involved include the infratemporal space, anterior zygomatic space, anterior masseter space, and cheek space [6,7]. The sutures seen in the research group most often passed through the temporal space, the anterior masseter space, and the cheek space, and occasionally passed through the anterior zygomatic space. When dissecting those criss-cross fibrotic scars, it is necessary to avoid damaging the branches of the facial nerve. In addition, the most difficult and risky step is to undermine the anterior masseter space and the buccal space. In fact, the author has no other "magic skills" except for comprehensively and systematically informing patients and their families of surgical risks and technical difficulties before surgery. Therefore, the only approach is to inject tumescent fluid into the scar and then carefully dissect it with ophthalmic scissors. Dissecting the branches of the facial nerve in the scar is an unprecedented challenge of the SMAS flap dissection. The author's team is studying preoperative diagnosis techniques, so it can be inferred that in the future there will be a new method of dissecting the scar while securing the facial nerve branches. In fact, the way of suture insertion is correct only when it passes through the subcutaneous fat layer. Doctors familiar with facial anatomy should know that other anatomical hierarchies are either ineffective, cannot accommodate these sutures, or damage important structures.

\section{A dilemma between pursuing a better outcome and not producing permanent facial paralysis}

This situation refers to the facts as follows. Firstly, the facial nerve branches are too thin (mostly the branches of the buccal fat pad area). Secondly, the scar texture is tough. Thirdly, the scar is large and involves multiple branches of the facial nerve (Fig. 11). In this scenario, the surgeon might choose to continue the dissection to achieve the expected outcome and so as to balance the symmetry of each side, including improving 
the dynamic deformity caused by scar adhesion; or to stop the dissection because of the extremely high risk of damaging the small and fragile facial nerve branches which may be cut off accidentally at any time in the scar. The latter option is undoubtedly safe, but a prominent problem has arisen poor lifting effect, asymmetry (related to scar asymmetry, adhesion asymmetry, and blindness operation), and the dynamic adhesion deformity remain unsolved. Consequently, this is a really tough and hopeless choice. The conclusions of this study are as follows.

\section{Conclusion}

The scars produced by facial liposuction and thread lift can be located at two hierarchies, namely, the subcutaneous tissue of the facial soft tissue and the facial space. Because SMAS is very fragile, it can be removed during liposuction. The scars caused by this can be distributed on the masseter fascia containing the branches of the facial nerve and the buccal fat pad capsule.

The surgeons must acquire a very proficient level of knowledge of facial nerve branch anatomy especially for the postliposuction and the post-thread-lift patients. Even the anatomical mutations in some individuals must be grasped.

\section{Conflicts of interest}

The authors have nothing to disclose.

\section{References}

1. Barton FE Jr. The "high SMAS" face lift technique. Aesthet Surg J 2002;22:481-6.

2. Wang ZJ, Bai C, Li G, Wang Y, Teng X, Gao J. [Compartmentalization and local structural anatomy of the face and neck: facial layers anatomy]. Chin J Aesthet Plast Surg 2020;31:12833. Chinese.

3. Zhang C, Wu L, Wang J. [Depressor orbicularis lateralis: concept, anatomy and clinical relevance]. Chin J Plast Surg 2018;34:977-80. Chinese.

4. Wang ZJ, Gao J, Li J. [Study on the anatomy of superficial musculo aponeurotic system related to rhytidectomy]. Chin J Pract Aesthet Plast Surg 1992;3:127-30. Chinese.

5. Wang ZJ, Wang N, Hu G, Zhang C, Wang J, Gao J. [The anatomy study and clinical signification of the branches of the facial nerve out of the parotid gland]. J Tissue Eng Reconstr Surg 2006;2:31-4. Chinese.

6. Aston SJ, Steinbrech DS, Walden JL. Aesthetic plastic surgery. Li J, Dai Q, Qiu K, translators. Beijing: Peking University Medical Press; 2012. p. 57-8. Chinese.

7. Mendelson BC, Jacobson SR. Surgical anatomy of the midcheek: facial layers, spaces, and the midcheek segments. Clin Plast Surg 2008;35:395-404; discussion 393.

8. Wang ZJ, Ma X, Yang L, Wang Y, Chen L, Lv H. High SMAS face lift: clinical experience. J Cosmet Med 2017;1:112-9.

9. Alpert BS. High SMAS face lift. In: Frame JD, Bagheri SC, Smith DJ Jr, Khan HA, editors. Aesthetic surgery techniques: a case-based approach. Edinburgh: Elsevier; 2019. p. 81-9.

10. Guyuron B, Seyed Forootan NS, Katira K. The super-high SMAS facelift technique with tailor tack plication. Aesthetic Plast Surg 2018;42:1531-9.

11. Wang ZJ, Wang Y, Shi H, Fan W, Dang N, Ma X, et al. [Key points of high SMAS facelift]. Chin J Plast Surg 2018;34:809-13. Chinese.

12. Yang N, Wang Z, Wang B, Su X, Lu N. [Anatomic study of malar fat pad and aging analysis]. Chin J Plast Surg 2012;5:28:2127. Chinese.

13. Swift A, Remington K. BeautiPHIcation ${ }^{\mathrm{TM}}$ : a global approach to facial beauty. Clin Plast Surg 2011;38:347-77.

14. Wang ZJ, Bai C, Li G, Teng X, Yang L, Li Y, et al. [Compartmentalization and local structural anatomy of the face and neck including the midface area, buccal fat pad, and modiolus]. Chin J Aesthet Plast Surg 2019;30:640-6. Chinese.

15. Wang ZJ. [Compartmentalization and local structural anatomy of the face and neck: superficial musculoaponeurotic system]. Chin J Aesthet Plast Surg 2020;31:531-4. Chinese.

16. Wang ZJ, Wang N, Tian H, Zhang C, Gao J. [Mixed tumor of parotid gland excised by retrograde dissection of facial nerve under full exposure]. Chin J Aesthet Plast Surg 2010;21:464-7. Chinese. 